\title{
Tailored magnetic and magnetoelectric response of polymer-based composites
}

P. Martins ${ }^{1, *}, Y$ Y. V. Kolen 'ko', J. Rivas ${ }^{2,3}$, and S. Lanceros-Mendez ${ }^{1, *}$

${ }^{1}$ Centro/Departamento de Física, Universidade do Minho, 4710-057 Braga, Portugal.

${ }^{2}$ International Iberian Nanotechnology Laboratory, Av. Mestre José Veiga, 4715-330 Braga, Portugal

${ }^{3}$ Centro/Departamento de Química Universidade do Minho, 4710-057 Braga, Portugal.

Nanomag Laboratory, Department of Applied Physics, Technological Research Institute

University of Santiago de Compostela. 15782 Santiago de Compostela, Spain

KEYWORDS: magnetoelectrics, nanoparticles, polymer-based, magnetostriction, multiferroic 


\section{ABSTRACT}

The manipulation of electric ordering with applied magnetic fields has been realized on magnetoelectric (ME) materials, however, their ME switching is often accompanied by significant hysteresis and coercivity that represents, for some applications, a severe weakness. To overcome this obstacle, this work focus on the development of a new type of ME polymer nanocomposites that exhibits tailored ME response at room temperature. The multiferroic nanocomposites are based on three different ferrite nanoparticles, $\mathrm{Zn}_{0.2} \mathrm{Mn}_{0.8} \mathrm{Fe}_{2} \mathrm{O}_{4}(\mathrm{ZMFO}), \mathrm{CoFe}_{2} \mathrm{O}_{4}$ (CFO) and $\mathrm{Fe}_{3} \mathrm{O}_{4}(\mathrm{FO})$, dispersed in a piezoelectric co-polymer poly(vinylindene fluoride-trifluoroethylene), $\mathrm{P}(\mathrm{VDF}-\mathrm{TrFE})$, matrix. No substantial differences were detected on the time-stable piezoelectric response of the composites $\left(\approx-28 \mathrm{pC} \cdot \mathrm{N}^{-1}\right)$ with distinct ferrite fillers and for the same ferrite content of 10wt.\%. Magnetic hysteresis loops from pure ferrite nanopowders showed different magnetic responses. ME results of the nanocomposite films with 10wt.\% ferrite content revealed that the ME induced voltage increases with increasing DC magnetic field until a maximum of 6.5 $\mathrm{mV} \cdot \mathrm{cm}^{-1} \cdot \mathrm{Oe}^{-1}$, at an optimum magnetic field of $0.26 \mathrm{~T}$, and $0.8 \mathrm{mV} \cdot \mathrm{cm}^{-1} \cdot \mathrm{Oe}^{-1}$, at an optimum magnetic field of $0.15 \mathrm{~T}$, for the $\mathrm{CFO} / \mathrm{P}(\mathrm{VDF}-\mathrm{TrFE})$ and $\mathrm{FO} / \mathrm{P}(\mathrm{VDF}-\mathrm{TrFE})$ composites, respectively. On the contrary, the ME response of the $\mathrm{ZMFO} / \mathrm{P}(\mathrm{VDF}-\mathrm{TrFE})$ exposed no hysteresis and high dependence on the ZMFO filler content. Possible innovative applications such as memories and information storage, signal processing, ME sensors and oscillators have been addressed for such ferrite/PVDF nanocomposites. 


\section{Introduction}

Magnetoelectric (ME) materials, characterized by the coupling between the magnetic and the electronic orders of matter, have drawn high interest due to the scientific and technological importance in performing a change of the electric polarization upon applying a magnetic field (direct ME effect) or to obtain a change of magnetization under an electric field (indirect ME effect) ${ }^{1-3}$. Two ways have been explored for this coupling: single-phase ME materials and the development of ME composites, in which magnetostrictive and piezoelectric materials are mechanically coupled ${ }^{4-6}$. Single-phase ME materials, also known as intrinsically ME materials, typically show a low temperature and small ME coupling, hindering in this way their incorporation into technological applications ${ }^{7-9}$. On the other hand, in the case of multiferroic composite materials with an extrinsically ME effect, the magnetic field induces a dimensional change in the magnetostrictive material that is transferred to the adjacent piezoelectric material, which in turn undergoes a mechanically induced change in its polarization ${ }^{4}$. This ME-product property leads to output signals at room temperature that are many orders of magnitude higher than in single-phase materials, making the products attractive for device application ${ }^{4}$. Such composite materials can be ceramic or polymer-based ${ }^{4,10}$. Although ceramic-based ME materials reveal ME coefficients three orders of magnitude higher than the ones present in polymer-based ME materials ${ }^{11-12}$, piezoelectric ceramics are limited by reactions at the interface regions, feature low electrical resistance and high dielectric losses, not allowing workable device applications ${ }^{3-4}$. Alternatively, polymer-based ME materials ${ }^{13}$ have attracted large interest since strain coupling typically does not deteriorate with operation, have a facile, cheap, low-temperature and scalable production methods compatible with industrial requirements 
are flexible structures without large leakage currents, can be fabricated into a variety of forms, such as thin sheets or molded shapes, can exhibit optimized tailored mechanical properties and in some cases biocompatibility ${ }^{3,14}$.

Two main types of polymer-based ME composites can be found in the literature: laminated composites and nanocomposites ${ }^{3}$. Despite the highest ME response of $\mathrm{P}(\mathrm{VDF}-\mathrm{TrFE}) / \mathrm{CoFe}_{2} \mathrm{O}_{4}$ nanocomposite ${ }^{15}\left(42 \mathrm{mV} \cdot \mathrm{cm}^{-1} \cdot \mathrm{Oe}^{-1}\right)$ being four orders of magnitude lower than the ME response $\left(383 \mathrm{~V} \cdot \mathrm{cm}^{-1} \cdot \mathrm{Oe}^{-1}\right)$ reported for $\mathrm{P}(\mathrm{VDF}-\mathrm{TrFE}) /$ Metglas $2605 \mathrm{SA} 1$ laminates ${ }^{16}$, its flexibility, simple fabrication, easy shaping, the possibilities of miniaturization and/or to produce large uniform areas and the absence of degradation at the piezoelectric/magnetostrictive interface are obvious advantages of nanocomposites ${ }^{3,16-17}$.

Recent results have demonstrated the potential applications of polymer-based ME nanocomposites in prototype devices ${ }^{3}$. In particular, strong efforts are being made to develop devices such as energy harvesters, transducers, actuators, memories, low temperature spintronics or magnetic sensors ${ }^{3,18-}$ 19. ME magnetic sensors have enormous potential as by-products related to magnetic sensors: electric current sensors, speed sensors, angular sensors, electronic steering, throttle control, battery management, vehicle transmission, digital compasses, and GPS devices are just some examples and many of them are already being studied ${ }^{3}$.

Nevertheless, non-negligible rate-independent memory effects (hysteresis) observed in most of the already developed polymer-based ME materials impact the performance of the final devices and could represent a severe drawback in their development ${ }^{20-22}$. Additionally the development of ME materials with no magnetic coercivity and hysteresis allow the development of sensors with low noise and high sensitivity, two fundamental requirements for their incorporation into technological devices $^{22-24}$. Thus, in order to enhance the application potential of polymer-based ME materials 
as magnetic sensors, there is a need to develop polymer-based ME materials with tailored magnetic hysteresis or coercivity ${ }^{20-24}$.

Herein, magnetostrictive $\mathrm{Zn}_{0.2} \mathrm{Mn}_{0.8} \mathrm{Fe}_{2} \mathrm{O}_{4}(\mathrm{ZMFO}), \mathrm{CoFe}_{2} \mathrm{O}_{4}(\mathrm{CFO})$ and $\mathrm{Fe}_{3} \mathrm{O}_{4}$ (FO) nanoparticles have been synthesized and introduced into a piezoelectric poly(vinylidenefluoride-cotrifluoroethylene) (PVDF-TrFE) matrix, aiming at obtaining polymer-based ME composite materials with tailored $\mathrm{ME}$ response. $\mathrm{ZMFO}$ and $\mathrm{FO}$ have been selected due to their magnetostriction values ( $\lambda=1$ and $25 \mathrm{ppm}$, respectively ${ }^{25-28}$ ) and absence of magnetic hysteresis and coercivity ${ }^{26,29-30}$. CFO $\left(\lambda=220 \mathrm{ppm}^{28}\right)$ was used in order to evaluate the influence of the magnetic hysteresis and coercivity in the ME response of the polymer-based composite. PVDFTrFE was used as the piezoelectric phase since it shows one of the highest piezoelectric responses among the small class of polymers that exhibit piezoelectricity ${ }^{3,22}$. Additionally, when crystallized from the melt this PVDF copolymer crystallizes in the piezoelectric phase, which is an essential factor for the preparation of ME composites ${ }^{14}$.

\section{Experimental}

Starting materials

$\mathrm{FeCl}_{3} \cdot 6 \mathrm{H}_{2} \mathrm{O}$ (99\%), $\mathrm{ZnCl}_{2}(98 \%), \mathrm{MnCl}_{2} \cdot 4 \mathrm{H}_{2} \mathrm{O}$ (99\%), $\mathrm{NiCl}_{2}(98 \%), \mathrm{CoCl}_{2} \cdot 6 \mathrm{H}_{2} \mathrm{O}(98 \%)$, $\mathrm{FeCl}_{2} \cdot 4 \mathrm{H}_{2} \mathrm{O}(99 \%)$, sodium oleate (82\%), aqueous $\mathrm{NH}_{4} \mathrm{OH}$ solution (28-30\%), and cyclohexane (99.8\%) were purchased from Sigma-Aldrich; ethanol (96\%) was purchased from Carlo Erba Reagents. $N, N$-Dimethylformamide (DMF, pure grade) was supplied by Fluka and P(VDF-TrFE) was supplied by Solvay Solexis. All chemicals were used as received without further purification. Ultrapure water was produced using a Milli-Q Advantage A10 system (Millipore). 
Synthesis of ferrite nanoparticles

The ZMFO, CFO and FO ferrite nanoparticles were synthesized using a hydrothermal method ${ }^{31}$. Briefly, $14 \mathrm{mmol}$ of $\mathrm{Fe}^{3+}$ precursor and $8 \mathrm{mmol}$ of $M^{2+}$ precursor $(M=\mathrm{Zn} / \mathrm{Mn}, \mathrm{Co}, \mathrm{Fe})$ were dissolved in $10 \mathrm{~mL}$ of Milli-Q water. A solution containing $0.5 \mathrm{~g}$ of sodium oleate dissolved in 10 $\mathrm{mL}$ of water was slowly added, following by a fast addition of $15 \mathrm{~mL}$ of concentrated ammonia solution. The hydrothermal treatment was performed at $200{ }^{\circ} \mathrm{C}$ for 1 day. The product of the treatment was collected by decantation, thoroughly washed with water and dried overnight at RT under reduced pressure $(\approx 0.08 \mathrm{MPa})$. The resulting dry powder was re-dispersed in cyclohexane and centrifuged at $3000 \mathrm{rpm}$ for $10 \mathrm{~min}$. Two products were obtained: a colloidal solution of small ferrite nanoparticles and precipitated large ferrite nanoparticles. The research described below is performed only using the latter. The centrifuged precipitate was dried at RT under reduced pressure and, finally, was homogenized in an agate mortar using a pestle.

\section{Composite preparation}

The desired amount of the magnetostrictive phase (ZMFO, CFO and FO) was added into DMF and placed in an ultrasound bath for $8 \mathrm{~h}$ in order to ensure a good dispersion of the nanoparticles. $\mathrm{P}(\mathrm{VDF}-\mathrm{TrFE})$ polymer was then added and mixed during 2 hours with the help of a mechanical Teflon stirrer in an ultrasound bath to avoid magnetic agglomeration during the mixing process. After that, the solution was spread on a clean glass substrate and solvent evaporation/samples crystallization was performed inside an oven at $210{ }^{\circ} \mathrm{C}$ for $10 \mathrm{~min}$. Polymer crystallization was stopped by cooling down films to room temperature. At the end of the process, the $\approx 50 \mu \mathrm{m}$ thick films were peeled from the glass substrate. Flexible ME composite films were thus prepared with $10 \%$ weigh content (wt.\%) of nanoparticles since obtaining films with good ME coupling and 
flexibility is expected with such a ferrite content ${ }^{22,32}$. Additionally ZMFO/P(VDF-TrFE) composite films with 20 and 50 wt.\% ferrite content were also prepared in order to evaluate the effect of the nanoparticle content in the piezoelectric, magnetic and ME response of the these composites.

\section{Materials Characterization}

Transmission electron microscopy (TEM) studies were performed using probe aberrationcorrected Titan ChemiSTEM (FEI) electron microscope, operated at $200 \mathrm{kV}$. The average size of nanoparticles was estimated using the Image $\mathbf{J}$ software by counting more than 350 nanoparticles. Powder X-ray diffraction (XRD) data were collected on a X'Pert PRO diffractometer (PANalytical) set at $45 \mathrm{kV}$ and $40 \mathrm{~mA}$, and equipped with $\mathrm{Cu} K \alpha$ radiation $(\lambda=1.541874 \AA$ ) and a PIXcel detector. Poling of the membranes was achieved, after an optimization procedure, by corona poling at $10 \mathrm{kV}$ during $120 \mathrm{~min}$ at $120{ }^{\circ} \mathrm{C}$ in a home-made chamber and cooling down to room temperature under applied electric field. The piezoelectric response $\left(d_{33}\right)$ of the samples was analyzed with a wide range $\mathrm{d}_{33}$-meter (model 8000, APC Int Ltd).

Magnetic hysteresis loops were measured at room temperature using an ADE 3473-70 Technologies vibrating sample magnetometer (VSM).

In order to obtain the out-of-plane ME coefficient $\alpha_{33}$ (the first index indicating the collinear ferroelectric poling and electrical measurement directions and the second indicating the applied magnetic field direction), a DC and AC magnetic field were applied along the direction of the electric polarization of the composites, i.e., perpendicular to the surface. 
The AC driving magnetic field of 1 Oe amplitude at $\approx 6 \mathrm{kHz}$ (resonance of the composite) was provided by a pair of Helmholtz coils and the DC field with a maximum value of $0.5 \mathrm{~T}$ was applied by an electromagnet.

The resonance frequency $\left(f_{r}\right)$ of the composites was determined by equation 1:

$$
f_{r}=\frac{n}{2 l} \sqrt{E_{Y} / \rho}
$$

where $n, E_{Y}$ and $\rho$ are the harmonic mode order, in-plane Young's modulus and density of the composites, respectively.

The induced ME voltage was measured with a Standford Research Lock-in amplifier (SR530).

Circular $1.4 \mathrm{~mm}$-diameter gold electrodes were sputtered on opposite sides of the samples prior to the ME characterization.

\section{RESULTS AND DISCUSSION}

In order to study the morphology and size of nanoparticles, TEM images and XRD patterns of the ferrite nanoparticles are shown in Figure 1. The average diameter of the nanoparticles was obtained from TEM images. 
a)

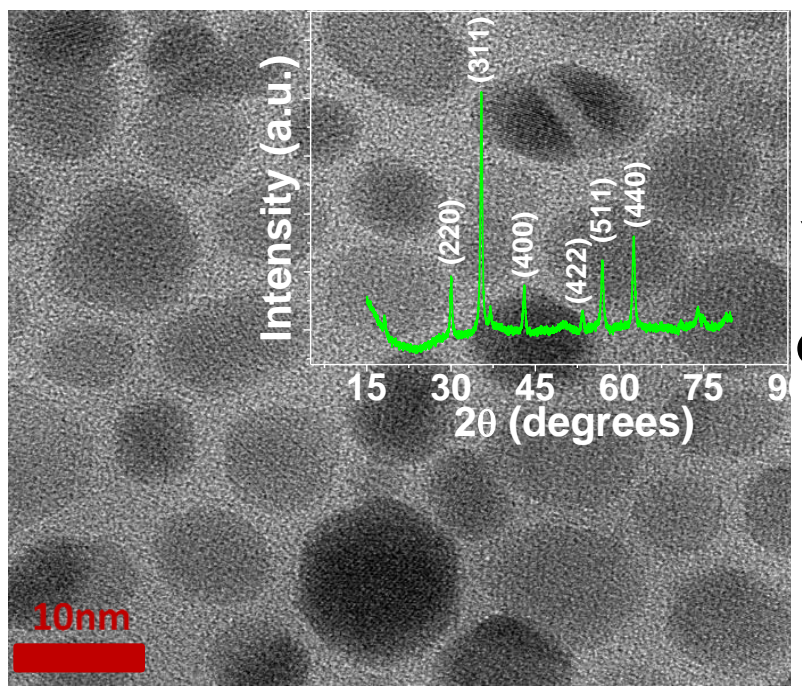

c)

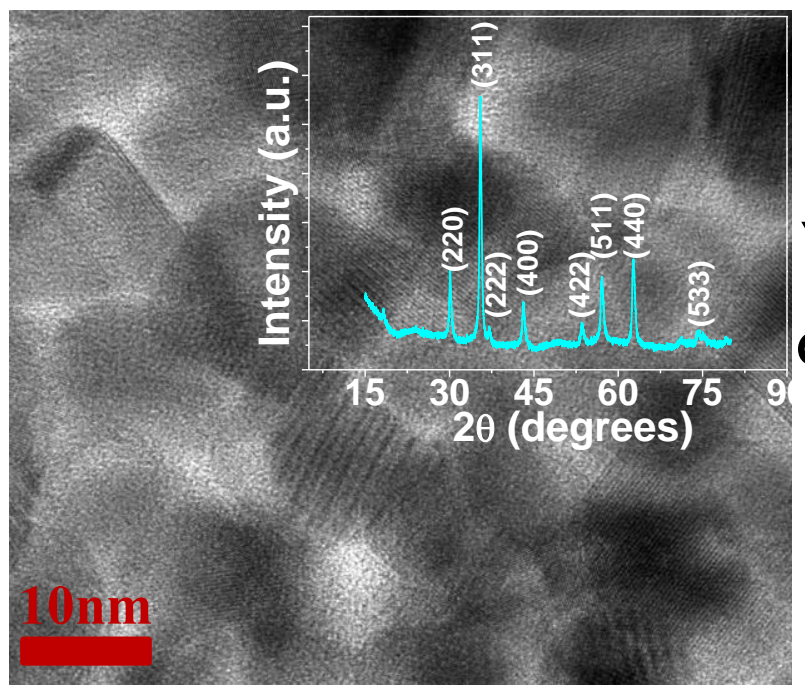

b)

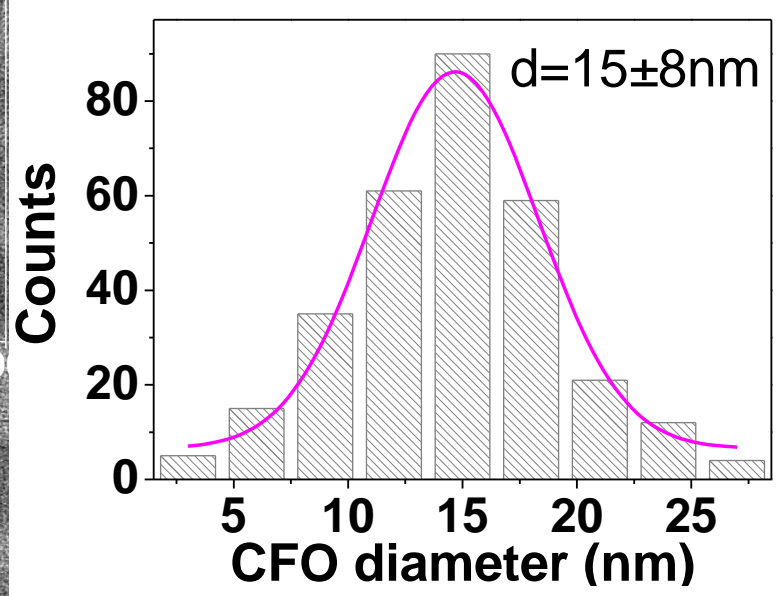

d)

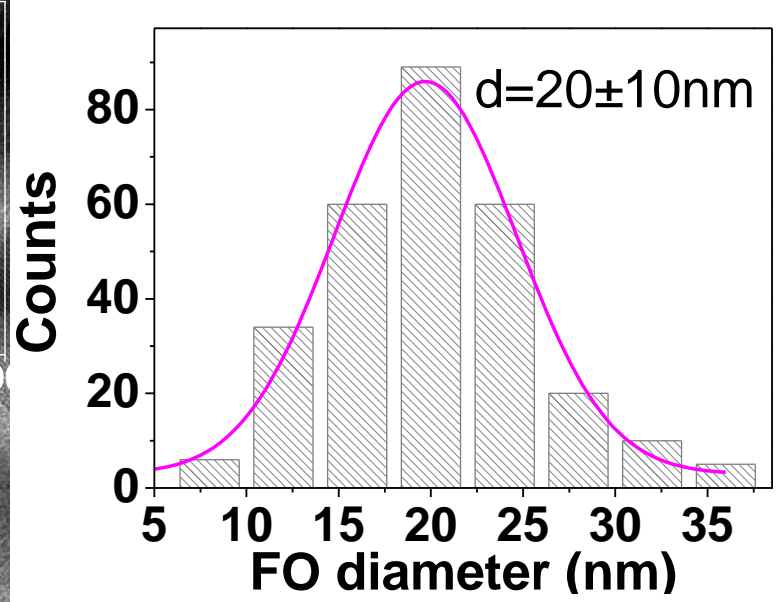


e)

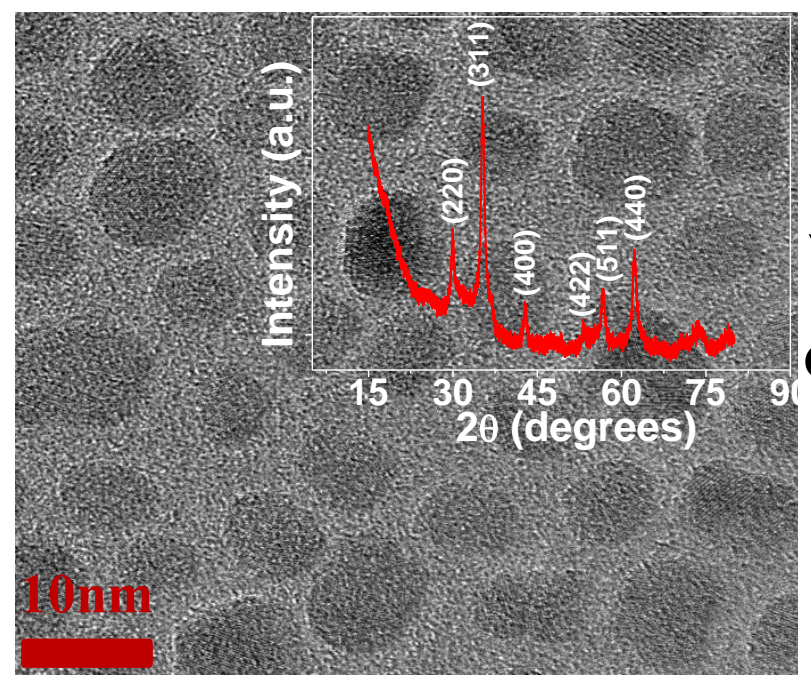

f)

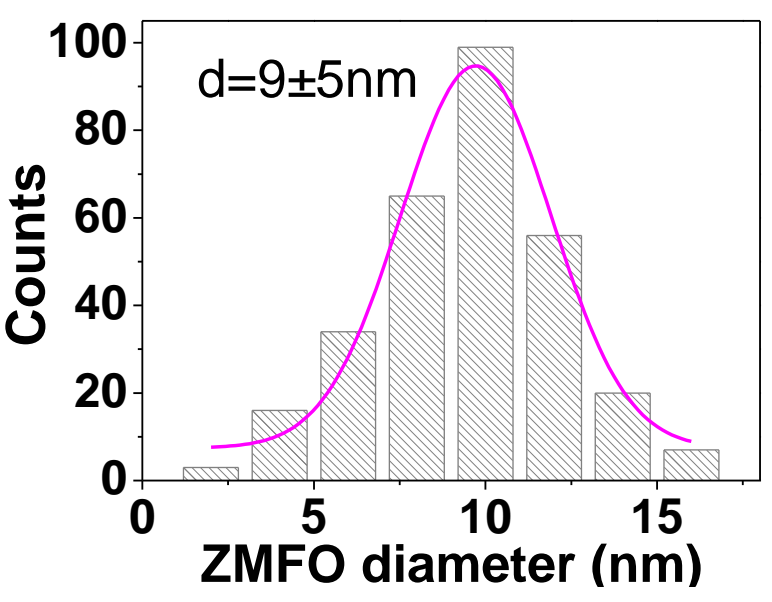

Figure 1. a) TEM image of CFO nanoparticles; b) size distribution of CFO nanoparticles; c) TEM image of FO nanoparticles; d) size distribution of FO nanoparticles; e) TEM image of ZMFO nanoparticles; and f) size distribution of ZMFO nanoparticles. Insets: corresponding Xray diffractions patters for each nanoparticle.

TEM images show a spherical shape of all the synthesized nanoparticles with average diameters of 9, 15 and $20 \mathrm{~nm}$ for the ZMFO, CFO and FO and ferrites, respectively.

Since the size of both ZMFO and FO nanoparticles is below the superparamagnetic limit $\left(25 \mathrm{~nm}^{33}\right)$ and that of CFO is above the superparamagnetic limit $\left(10 \mathrm{~nm}^{33}\right)$, superparamagnetic behavior should be just observed in the ZMFO/P(VDF-TrFE) and FO/P(VDF-TrFE) nanocomposites. Additionally, the size range found on the nanoparticles have no influence on the mechanical and ME response of the nanocomposites ${ }^{15,34}$.

In the XRD patterns of all ferrite nanoparticles no peaks corresponding to impurities were detected. Additionally, the narrow sharp peaks reveal the high purity of the synthesized ferrites ${ }^{35}$. All the ferrites have inverse cubic spinel structure with space group $F d-3 m^{36}$. 
In the ferrite/P(VDF-TrFE) composites, the ME effect is generated as a product property between magnetostrictive and piezoelectric phases and, therefore, a high piezoelectric coefficient of the polymer matrix will favor a large ME coupling ${ }^{37}$. The variations of the modulus (the $\mathrm{d}_{33}$ is negative for PVDF and copolymers) of the piezoelectric $\left|\mathrm{d}_{33}\right|$ coefficient with the ferrite type and content as well as it stability over time is represented in Figure 2.

a)

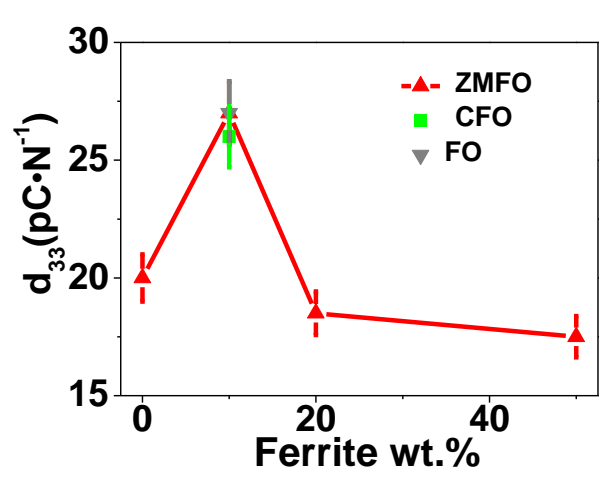

b)

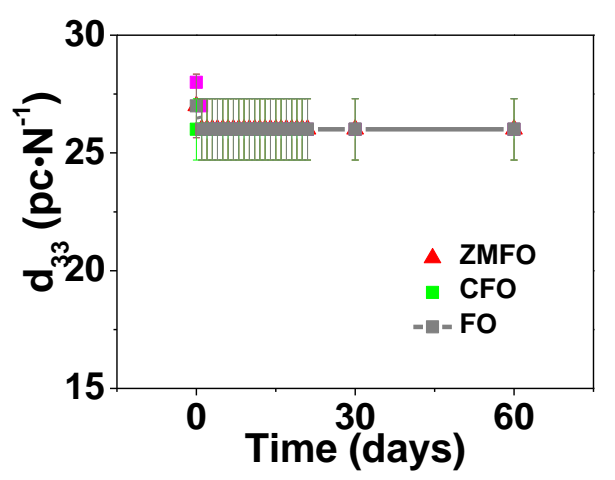

Figure 2. a) Variation of the ferrite/P(VDF-TrFE) $\left|\mathrm{d}_{33}\right|$ value with ferrite type and ZMFO content; b) Evolution of the ferrite/P(VDF-TrFE) $\left|\mathrm{d}_{33}\right|$ coefficient over time.

Figure 2a shows the correlation between the piezoelectric response of the composites and the ferrite type and content. No substantial differences are detected in the piezoelectric properties of composites with distinct ferrite fillers for a given ferrite content (10wt.\%). This is expected as the piezoelectricity is fully ascribed to the polymer. As ZMFO ferrite concentration increases, the 
piezoelectric response increases until a maximum value $\approx 28 \mathrm{pC} \mathrm{N}^{-1}$ at a concentration of $10 \mathrm{wt} \%$ ferrite content. For higher concentrations, the piezoelectricity decrease to values $\approx 18 \mathrm{pC} \mathrm{N} \mathrm{N}^{-1}$ (similar behavior was found for the other ferrite composites). The presence of the ferrite nanoparticles (until $10 \mathrm{wt} . \%$ ) improves the piezoelectric properties of the composites due to strong electrostatic interactions between nanoparticles and polymer at the interface regions with strong coupling and improved transduction properties ${ }^{38-39}$. Higher contents of ferrite nanoparticles decrease the piezoelectricity of the composite due to a slight disruption of the connectivity of the polymer matrix related also with the existence of particle agglomerates ${ }^{22,32}$. Figure $\mathbf{2 b}$ reveals that the piezoelectric response of all composites is stable over time until at least two months.

With respect to the magnetic properties, Figure 3 shows the magnetization loops of the different ferrite nanoparticles and for all composites with 10wt.\% ferrite content.

a)

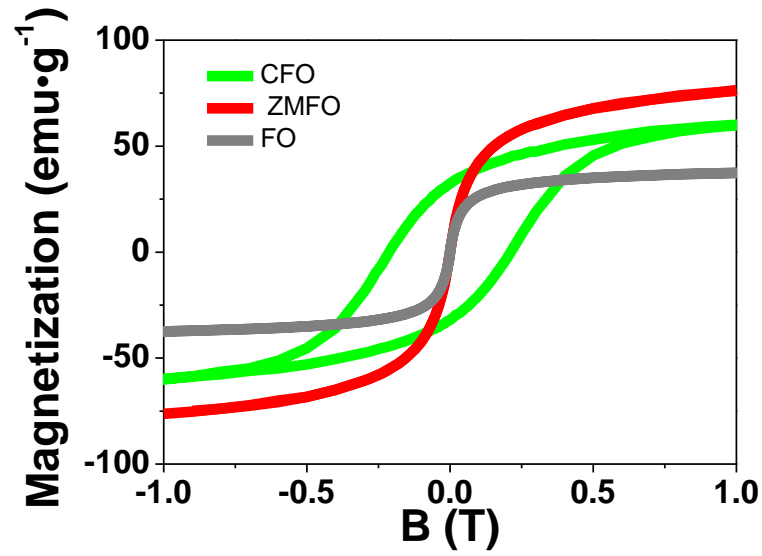

b)

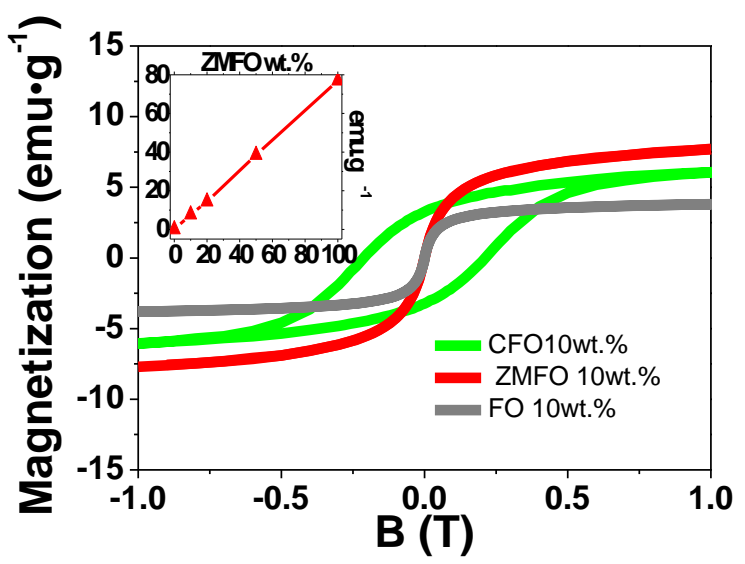

Figure 3. a) Room temperature hysteresis loops for the pure ferrite nanoparticle powders; b) Room temperature hysteresis loops for the ferrite/P(VDF-TrFE) composites with 10 wt.\% ferrite content.

Hysteresis loops of the pure ferrite nanopowders reveal the expected distinct magnetic behaviors (Figure 3a) of the different nanoparticles: while CFO develops a hysteresis loop with coercivity 
of $\approx 0.25 \mathrm{~T}, 30 \mathrm{emu} \cdot \mathrm{g}^{-1}$ remanence and reaches a maximum magnetization of $61 \mathrm{emu} \cdot \mathrm{g}^{-1}$ at a $1 \mathrm{~T}$ applied magnetic field, ZMFO and FO show almost a complete absence of hysteresis, remanence and coercivity. For these two ferrites, room temperature is above the blocking temperature and the magnetic moment of the particle is free to rotate in response to the applied magnetic field ${ }^{15,40}$, resulting in the magnetization at $1 \mathrm{~T} 77 \mathrm{emu} \cdot \mathrm{g}^{-1}$ and $38 \mathrm{emu} \cdot \mathrm{g}^{-1}$ for the ZMFO and FO powders, respectively. The shape and maximum magnetization values of the measured hysteresis loops for the multiferroic composite samples (Figure 3b) demonstrate that magnetic particles are randomly oriented within the polymer matrix ${ }^{15}$. Additionally, the maximum magnetization of $3.8,6.1$ and $7.7 \mathrm{emu}^{-1} \mathrm{~g}^{-1}$ found for $\mathrm{FO} / \mathrm{P}(\mathrm{VDF}-\mathrm{TrFE}), \quad \mathrm{CFO} / \mathrm{P}(\mathrm{VDF}-\mathrm{TrFE})$ and $\mathrm{ZMFO} / \mathrm{P}(\mathrm{VDF}-\mathrm{TrFE})$ composites, respectively, with $10 \mathrm{wt} . \%$ ferrite content reveal that nanoparticles are well distributed and dispersed and that maximum magnetization value is directly proportional to the amount of nanoparticles inside the polymer-based composite ${ }^{15,32,39}$. The inset in Figure 3b shows thus a linear increase in the magnetic response with increasing ZMFO content.

Figure 4a shows the variation of the ME voltage coefficient with the DC magnetic field for the different ferrite/P(VDF-TrFE) composites with $10 \mathrm{wt} . \%$ ferrite content, measured under an AC field of $1 \mathrm{Oe}$ at $6 \mathrm{kHz}$ frequency.

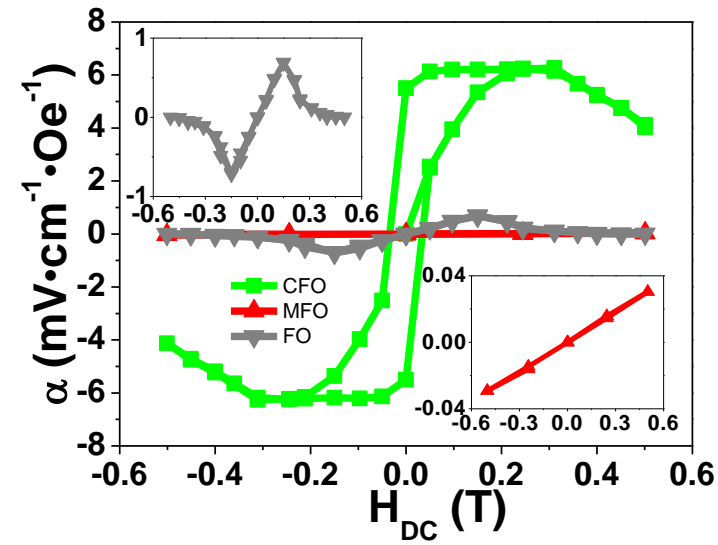

a)

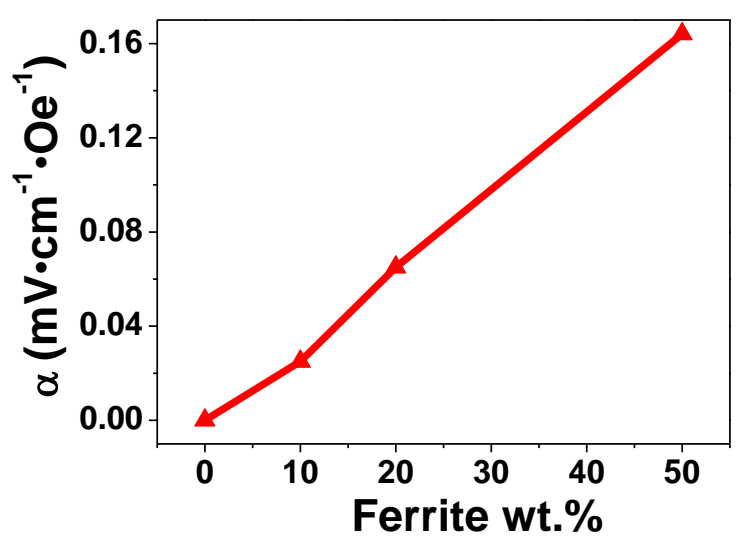

b) 
Figure 4. a) ME coefficients as a function of the bias field for the ferrite/P(VDF-TrFE) composites with 10 wt.\% ferrite content. Insets are magnifications of the ZMFO and FO composite responses; b) ME coefficient as a function of the ZMFO ferrite content.

It can be observed that the induced voltage increases with increasing DC magnetic field until a maximum of $6.5 \mathrm{mV} \cdot \mathrm{cm}^{-1} \cdot \mathrm{Oe}^{-1}$ (at an optimum magnetic field of $0.26 \mathrm{~T}$ ) and $0.8 \mathrm{mV} \cdot \mathrm{cm}^{-1} \cdot \mathrm{Oe}^{-1}$ (at an optimum magnetic field of $0.15 \mathrm{~T}$ ) for the CFO/P(VDF-TrFE) and FO/P(VDF-TrFE) composites, respectively. Such behavior is explained by the increase of the effective piezomagnetic coefficient until the optimum DC magnetic field is reached. With further increase in the DC magnetic field, a decrease in the induced voltage is observed for both composites resulting from the saturation of the magnetostriction coefficient ${ }^{41-43}$. Additionally, the ME response found for the $\mathrm{CFO} / \mathrm{P}(\mathrm{VDF}-\mathrm{TrFE})$ composites show an hysteretic behavior related to the magnetic hysteresis observed in Figure 3.

On the other hand, the ME response of the ZMFO/P(VDF-TrFE) composite shows a linear behavior as a consequence of the increasing piezomagnetic behavior with increasing DC magnetic field and due to the unsaturated magnetostriction until $0.5 \mathrm{~T}^{25}$.

Figure 4b shows the ME response of the ZMFO/P(VDF-TrFE) nanocomposites at a bias field of 0.5 $\mathrm{T}$ with increasing ferrite loading. The linear increase in the ME voltage is explained by the increase in the magnetostriction related to the increase of the magnetostrictive phase ${ }^{32}$.

Whereas in memory applications and information storage ME hysteresis found on CFO/P(VDFTrFE) composites allows for non-volatility, in devices such as ME sensors and oscillators hysteresis is responsible for losses and gives rise to detrimental side effects such as low precision, drift and asymmetric oscillation ${ }^{21}$. In this way, the linear, non-hysteretic ME response and the $4 \mu \mathrm{V} / \mathrm{Oe}$ magnetic field sensitivity found for $\mathrm{ZMFO} / \mathrm{P}(\mathrm{VDF}-\mathrm{TrFE})$ composites allows to obtain 
sensors and oscillators with low noise and high sensibility, two fundamental requirements in order to allow their incorporation into devices ${ }^{23-24,44}$.

Recently, there has been interest in using the non-linear ME effect, such as the one found on FO/P(VDF-TrFE) composites, on signal processing. For example, non-linear ME interactions allow the realization of active mode ME sensors which can modulate weak low-frequency signals to a higher frequency bandwidth ${ }^{45}$.

Since the ME effect in polymer composites can be suitably described by ${ }^{46-47}$;

$$
\alpha_{33}=(1-\phi) \frac{L_{E}}{\varepsilon}\left(d_{31 p} \frac{d Y_{x p}}{d H_{m}}+d_{32 p} \frac{d T_{y p}}{d H_{m}}+d_{33 p} \frac{d T_{z p}}{d H_{M}}\right)\left(\frac{d H_{m}}{d H}\right)
$$

where $L_{E}$ and $\frac{d H_{m}}{d H}$ are given by:

$$
\begin{aligned}
& L_{E}=\frac{\left.\left\lfloor\varepsilon_{m}+2 \varepsilon_{p}\right]\right\rfloor}{\left[(1-\phi) \varepsilon_{m}+(2+\phi) \varepsilon_{p}\right]} \\
& \frac{d H_{m}}{d H}=\frac{3 \xi_{p}}{(1-\phi)\left(\xi_{m}+\frac{d M_{m}}{d H_{m}}\right)+(2+\phi) \xi_{p}}
\end{aligned}
$$

( $p$ and $\mathrm{m}$ indicating the piezoelectric and magnetostrictive phase respectively; $d_{3 n}$ the piezoelectric coefficients; $\varepsilon$ the dielectric constant, $\phi$ the volume fraction of the magnetostrictive component; $T$ and $H$ the stress and applied magnetic field, respectively; $\xi$ the magnetic permeability and $M$ the magnetization) the ME voltage coefficient, $\alpha_{33}$, in these composites can be further increased by increasing filler content, $\phi$, dielectric constant of the composite, $\varepsilon$, or by suitably modifying the elastic modulus ${ }^{48-50}$. 


\section{Conclusions}

Magnetostrictive ZMFO (9 $\mathrm{nm})$, CFO $(15 \mathrm{~nm})$ and FO (20 nm) nanoparticles have been synthesized using a hydrothermal method and introduced into a piezoelectric P(VDF-TrFE) matrix, obtaining multiferroic polymer nanocomposites produced by a simple solvent casting method.

Time-stable piezoelectric response of composites $\left(\approx-28 \mathrm{pC} \mathrm{N}^{-1}\right)$ was observed with distinct ferrite fillers and with the same ferrite content (10wt.\%).

While CFO presents hysteresis loop with coercivity of $\approx 0.25 \mathrm{~T}, 30 \mathrm{emu} \cdot \mathrm{g}^{-1}$ remanence and reaches a maximum magnetic moment of $61 \mathrm{emu} \cdot \mathrm{g}^{-1}$ at a $1 \mathrm{~T}$ applied magnetic field, the ZMFO and FO revealed no hysteresis, no remanence and no coercivity. The shape and magnetization maximum values of the measured hysteresis loops for the multiferroic composite are fully determined by the ferrite content and type. Composite films with $10 \mathrm{wt} \%$ of ferrite content showed that the ME coefficient increases with increasing DC magnetic field until a maximum of $6.5 \mathrm{mV} \cdot \mathrm{cm}^{-1} \cdot \mathrm{Oe}^{-1}$ and $0.8 \mathrm{mV} \cdot \mathrm{cm}^{-1} \cdot \mathrm{Oe}^{-1}$ for the $\mathrm{CFO} / \mathrm{P}(\mathrm{VDF}-\mathrm{TrFE})$ and $\mathrm{FO} / \mathrm{P}(\mathrm{VDF}-\mathrm{TrFE})$ composites, respectively. In contrast, the $\mathrm{ME}$ response of the $\mathrm{ZMFO} / \mathrm{P}(\mathrm{VDF}-\mathrm{TrFE})$ revealed a linear response, without hysteresis and proportional to the content of the ZMFO nanoparticles. Thus, the different properties of the developed ferrite/P(VDF-TrFE) composites fulfill a wide range of ME responses leading to potential incorporation into innovative technological devices.

\section{AUTHOR INFORMATION}

\section{Corresponding Author}

*P. Martins and S. Lanceros Mendez 
Centro/Departamento de Física, Universidade do Minho, 4710-057 Braga, Portugal.

E-mail: pmartins@fisica.uminho.pt; lanceros@fisica.uminho.pt

\section{Author Contributions}

The manuscript was written through contributions of all authors. All authors have given approval to the final version of the manuscript.

\section{Funding Sources}

FCT - Portuguese Foundation for Science and Technology

\section{ACKNOWLEDGMENT}

We thank Dr. E. Carbó-Argibay for his assistance with TEM analysis. This work is funded by FEDER funds through the "Programa Operacional Factores de Competitividade - COMPETE" and by national funds from FCT - Portuguese Foundation for Science and Technology in the framework of the strategic project Strategic Project PEST-C/FIS/UI607/2014. The authors also thank funding from Matepro -Optimizing Materials and Processes", ref. NORTE-07-0124FEDER-000037", co-funded by the "Programa Operacional Regional do Norte" (ON.2 - O Novo Norte), under the "Quadro de Referência Estratégico Nacional" (QREN), through the "Fundo Europeu de Desenvolvimento Regional" (FEDER). P. Martins acknowledges also support from FCT GRANT SFRH/BPD/96227/2013.

\footnotetext{
ABBREVIATIONS

ME, magnetoelectric; ZMFO, $\mathrm{Zn}_{0.2} \mathrm{Mn}_{0.8} \mathrm{Fe}_{2} \mathrm{O}_{4} ; \mathrm{CFO}, \mathrm{CoFe}_{2} \mathrm{O}_{4} ; \mathrm{FO}, \mathrm{Fe}_{3} \mathrm{O}_{4} ; \mathrm{P}(\mathrm{VDF}-\mathrm{TrFE})$, poly(vinylindene fluoride-trifluoroethylene); TEM, Transmission electron microscopy; XRD, powder X-ray diffraction; VSM, vibrating sample magnetometer.
} 


\section{REFERENCES}

1. Han, T. C.; Lee, Y. C.; Chu, Y. T. Effect of Cobalt Doping on Site-Disorder and Magnetic Behavior of Magnetoelectric GaFeO3 Nanoparticles Appl. Phys. Lett. 2014, 105, 212407.

2. Nguyen, T. H. L.; Laffont, L.; Capsal, J.-F.; Cottinet, P.-J.; Lonjon, A.; Dantras, E.; Lacabanne, C. Magnetoelectric Properties of Nickel Nanowires-P(VDF-TrFE) Composites Mater. Chem. Phys 2015, 153, 195-201.

3. Martins, P.; Lanceros-Méndez, S. Polymer-Based Magnetoelectric Materials Adv. Funct. Mater. 2013, 23, 3371-3385.

4. Kulkarni, A.; Meurisch, K.; Teliban, I.; Jahns, R.; Strunskus, T.; Piorra, A.; Knöchel, R.; Faupel, F. Giant Magnetoelectric Effect at Low Frequencies in Polymer-Based Thin Film Composites Appl. Phys. Lett. 2014, 104, 022904.

5. $\quad$ Martins, P.; Larrea, A.; Golçalves, R.; Botelho, G.; Ramana, E. V.; Mendiratta, S. K.; Sebastian, V.; Lanceros-Mendez, S. Novel Anisotropic Magnetoelectric Effect on $\Delta$ Feo(Oh)/P(Vdf-Trfe) Multiferroic Composites ACS Appl. Mater. Interfaces 2015, 7, $11224-$ 11229.

6. $\quad$ Li, D. Y.; Zeng, Y. J.; Batuk, D.; Pereira, L. M. C.; Ye, Z. Z.; Fleischmann, C.; Menghini, M.; Nikitenko, S.; Hadermann, J.; Temst, K.; Vantomme, A.; Van Bael, M. J.; Locquet, J. P.; Van Haesendonck, C. Relaxor Ferroelectricity and Magnetoelectric Coupling in Zno-Co Nanocomposite Thin Films: Beyond Multiferroic Composites ACS Appl. Mater. Interfaces 2014, 6, 4737-4742.

7. Yang, T. N.; Hu, J. M.; Nan, C. W.; Chen, L. Q. Predicting Effective Magnetoelectric Response in Magnetic-Ferroelectric Composites Via Phase-Field Modeling Appl. Phys. Lett. 2014, 104, 052904.

8. Zuo, Z. J.; Pan, D. A.; Lu, J.; Zhang, S. G.; Tian, J. J.; Qiao, L. J.; Volinsky, A. A. Multiplied Magnetoelectric Effect in Multi-Faceted Magnetoelectric Composite Appl. Phys. Lett. 2014, 104, 032906.

9. $\quad$ Kohiki, S.; Okada, K.; Mitome, M.; Kohno, A.; Kinoshita, T.; Iyama, K.; Tsunawaki, F.; Deguchi, H. Magnetic and Magnetoelectric Properties of Self-Assembled Fe 2.5mn 0.5o 4 Nanocrystals ACS Appl. Mater. Interfaces 2011, 3, 3589-3593.

10. Zhang, J.; Li, P.; Wen, Y.; He, W.; Yang, A.; Lu, C. Giant Self-Biased Magnetoelectric Response with Obvious Hysteresis in Layered Homogeneous Composites of Negative Magnetostrictive Material Samfenol and Piezoelectric Ceramics Appl. Phys. Lett. 2013, 103, 202902.

11. Kulawik, J.; Szwagierczak, D.; Guzdek, P. Magnetic, Magnetoelectric and Dielectric Behavior of Cofe 2o 4- $\mathrm{Pb}(\mathrm{Fe} 1 / 2 \mathrm{nb} 1 / 2) \mathrm{O} 3$ Particulate and Layered Composites Journal of Magnetism and Magnetic Materials 2012, 324, 3052-3057.

12. Guzdek, P. The Magnetostrictive and Magnetoelectric Characterization of Ni 0.3zn0.62 cu0.08fe2o 4-Pb(Fenb)0.5o3 Laminated Composite Journal of Magnetism and Magnetic Materials 2014, 349, 219-223.

13. Szklarska-ŁUkasik, M.; Guzdek, P.; Dudek, M.; Pawlaczyk, A.; Chmist, J.; Dorowski, W.; Pszczoła, J. Magnetoelectric Properties of Tb0.27-Xdy0.73-Yy X+Yfe2/Pvdf Composites Journal of Alloys and Compounds 2013, 549, 276-282. 
14. Martins, P.; Lopes, A. C.; Lanceros-Mendez, S. Electroactive Phases of Poly(Vinylidene Fluoride): Determination, Processing and Applications Prog. Polym. Sci. 2014, 39, 683-706.

15. Martins, P.; Gonçalves, R.; Lanceros-Mendez, S.; Lasheras, A.; Gutiérrez, J.;

Barandiarán, J. M. Effect of Filler Dispersion and Dispersion Method on the Piezoelectric and Magnetoelectric Response of Cofe2o4/P(Vdf-Trfe) Nanocomposites Appl. Surf. Sci. 2014, 313, 215-219.

16. Jin, J.; Lu, S. G.; Chanthad, C.; Zhang, Q.; Haque, M. A.; Wang, Q. Multiferroic Polymer Composites with Greatly Enhanced Magnetoelectric Effect under a Low Magnetic Bias Adv. Mater. 2011, 23, 3853-3858.

17. Silva, M.; Reis, S.; Lehmann, C. S.; Martins, P.; Lanceros-Mendez, S.; Lasheras, A.; Gutiérrez, J.; Barandiarán, J. M. Optimization of the Magnetoelectric Response of Poly(Vinylidene Fluoride)/Epoxy/Vitrovac Laminates ACS Appl. Mater. Interfaces 2013, 5, 10912-10919.

18. Fusil, S.; Garcia, V.; Barthélémy, A.; Bibes, M., Magnetoelectric Devices for Spintronics. In Annual Review of Materials Research, 2014; Vol. 44, pp 91-116.

19. Phuoc, N. N.; Ong, C. K. Electric Field Modulation of Ultra-High Resonance Frequency in Obliquely Deposited [Pb(Mg1/3nb2/3)O3]0.68-[Pbtio3] 0.32(011)/Fecozr Heterostructure for Reconfigurable Magnetoelectric Microwave Devices Appl. Phys. Lett. 2014, 105, 022905.

20. Davino, D.; Visone, C.; Ambrosino, C.; Campopiano, S.; Cusano, A.; Cutolo, A. Compensation of Hysteresis in Magnetic Field Sensors Employing Fiber Bragg Grating and Magneto-Elastic Materials Sens. Actuator A-Phys. 2008, 147, 127-136.

21. Oh, Y. S.; Artyukhin, S.; Yang, J. J.; Zapf, V.; Kim, J. W.; Vanderbilt, D.; Cheong, S. W. Non-Hysteretic Colossal Magnetoelectricity in a Collinear Antiferromagnet Nat. Commun. 2014, 5,3201 .

22. Martins, P.; Moya, X.; Phillips, L. C.; Kar-Narayan, S.; Mathur, N. D.; LancerosMendez, S. Linear Anhysteretic Direct Magnetoelectric Effect in Ni

0.5zn0.5 fe2o4/Poly(Vinylidene Fluoride-Trifluoroethylene) 0-3 Nanocomposites J. Phys. D: Appl. Phys. 2011, 44, 482001.

23. Pong, P. W. T.; Schrag, B.; Shapiro, A. J.; McMichael, R. D.; Egelhoff, W. F. Hysteresis Loop Collapse for Linear Response in Magnetic-Tunnel-Junction Sensors J. Appl. Phys. 2009, 105, $07 \mathrm{E} 723$.

24. Yin, X.; Skomski, R.; Sellmyer, D.; Liou, S.-H.; Russek, S. E.; Evarts, E. R.; Moreland, J.; Edelstein, A. S.; Yuan, L.; Yan, M. L.; Shen, J. Adjusting Magnetic Nanostructures for HighPerformance Magnetic Sensors J. Appl. Phys. 2014, 115, 17 E528.

25. Bieńkowski, A.; Szewczyk, R.; Wiśniewska, A. Magnetostrictive Properties and Magnetoelastic Villari Effect in the High-Permeability Mn-Zn Ferrites Czech J. Phys. 2004, 54, D169-D172.

26. Bozorth, R. M.; Tilden, E. F.; Williams, A. J. Anisotropy and Magnetostriction of Some Ferrites Phys. Rev. 1955, 99, 1788-1798.

27. Odkhuu, D.; Taivansaikhan, P.; Yun, W. S.; Hong, S. C. A First-Principles Study of Magnetostrictions of Fe3o 4 and Cofe2o4 J. Appl. Phys. 2014, 115, 17 A916.

28. Fritsch, D.; Ederer, C. Epitaxial Strain Effects in the Spinel Ferrites Cofe2 O4 and Nife2 O4 from First Principles Phys. Rev. B 2010, 82, 104117.

29. Nabiyouni, G.; Julaee, M.; Ghanbari, D.; Aliabadi, P. C.; Safaie, N. Room Temperature Synthesis and Magnetic Property Studies of Fe3o4 Nanoparticles Prepared by a Simple Precipitation Method Journal of Industrial and Engineering Chemistry 2015, 21, 599-603. 
30. Wang, J.; Zeng, C.; Peng, Z.; Chen, Q. Synthesis and Magnetic Properties of Zn1-Xmnxfe2o4 Nanoparticles Physica B: Condensed Matter 2004, 349, 124-128.

31. Kolen'Ko, Y. V.; Bañobre-López, M.; Rodríguez-Abreu, C.; Carbó-Argibay, E.; Sailsman, A.; Piñeiro-Redondo, Y.; Cerqueira, M. F.; Petrovykh, D. Y.; Kovnir, K.; Lebedev, O. I.; Rivas, J. Large-Scale Synthesis of Colloidal Fe3o4 Nanoparticles Exhibiting High Heating Efficiency in Magnetic Hyperthermia J. Phys. Chem. C 2014, 118, 8691-8701.

32. Martins, P.; Lasheras, A.; Gutierrez, J.; Barandiaran, J. M.; Orue, I.; Lanceros-Mendez, S. Optimizing Piezoelectric and Magnetoelectric Responses on Cofe 2o4/P(Vdf-Trfe) Nanocomposites J. Phys. D: Appl. Phys. 2011, 44, 495303.

33. Krishnan, K. M.; Pakhomov, A. B.; Bao, Y.; Blomqvist, P.; Chun, Y.; Gonzales, M.; Griffin, K.; Ji, X.; Roberts, B. K. Nanomagnetism and Spin Electronics: Materials, Microstructure and Novel Properties J. Mater. Sci. 2006, 41, 793-815.

34. Choi, J.; Shin, H.; Yang, S.; Cho, M. The Influence of Nanoparticle Size on the Mechanical Properties of Polymer Nanocomposites and the Associated Interphase Region: A Multiscale Approach Compos. Struct. 2015, 119, 365-376.

35. Liu, X.-D.; Chen, H.; Liu, S.-S.; Ye, L.-Q.; Li, Y.-P. Hydrothermal Synthesis of Superparamagnetic Fe3o4 Nanoparticles with Ionic Liquids as Stabilizer Mater. Res. Bull. 2015, $62,217-221$.

36. Houshiar, M.; Zebhi, F.; Razi, Z. J.; Alidoust, A.; Askari, Z. Synthesis of Cobalt Ferrite (Cofe2o4) Nanoparticles Using Combustion, Coprecipitation, and Precipitation Methods: A Comparison Study of Size, Structural, and Magnetic Properties J. Magn. Magn. Mater. 2014, 371, 43-48.

37. Nan, C. W.; Bichurin, M. I.; Dong, S.; Viehland, D.; Srinivasan, G. Multiferroic Magnetoelectric Composites: Historical Perspective, Status, and Future Directions J. Appl. Phys. 2008, 103.

38. Liu, X.; Liu, S.; Han, M. G.; Zhao, L.; Deng, H.; Li, J.; Zhu, Y.; Krusin-Elbaum, L.; O'Brien, S. Magnetoelectricity in Cofe2o4 Nanocrystal-P(Vdf-Hfp) Thin Films Nanoscale Res. Lett. 2013, 8, 1-10.

39. Goncalvez, R.; Martins, P.; Correia, D.; Sencadas, V.; Vilas, J. L.; Leon, L. M.; Botelho, G. L.; lanceros-mendez, s. Development of Novel Magnetoelectric Cofe2o4 /Poly(Vinylidene Fluoride) Microspheres RSC Adv. 2015, 5, 35852-35857.

40. Martins, P.; Costa, C. M.; Botelho, G.; Lanceros-Mendez, S.; Barandiaran, J. M.; Gutierrez, J. Dielectric and Magnetic Properties of Ferrite/Poly(Vinylidene Fluoride) Nanocomposites Mater. Chem. Phys. 2012, 131, 698-705.

41. Dong, X. W.; Wang, B.; Wang, K. F.; Wan, J. G.; Liu, J. M. Ultra-Sensitive Detection of Magnetic Field and Its Direction Using Bilayer Pvdf/Metglas Laminate Sensors and Actuators, A: Physical 2009, 153, 64-68.

42. Koo, Y. S.; Song, K. M.; Hur, N.; Jung, J. H.; Jang, T. H.; Lee, H. J.; Koo, T. Y.; Jeong, Y. H.; Cho, J. H.; Jo, Y. H. Strain-Induced Magnetoelectric Coupling in Batio3 / Fe 3 O4 Core/Shell Nanoparticles Applied Physics Letters 2009, 94.

43. Zheng, Y. X.; Cao, Q. Q.; Zhang, C. L.; Xuan, H. C.; Wang, L. Y.; Wang, D. H.; Du, Y. W. Study of Uniaxial Magnetism and Enhanced Magnetostriction in Magnetic-Annealed Polycrystalline Cofe2o4 Journal of Applied Physics 2011, 110.

44. Zhang, L.; Or, S. W.; Leung, C. M. Voltage-Mode Direct-Current Magnetoelectric Sensor Based on Piezoelectric-Magnetostrictive Heterostructure Journal of Applied Physics 2015, 117. 
45. Shen, Y.; Gao, J.; Wang, Y.; Li, J.; Viehland, D. High Non-Linear Magnetoelectric Coefficient in Metglas/Pmn-Pt Laminate Composites under Zero Direct Current Magnetic Bias Journal of Applied Physics 2014, 115, 094102.

46. Wong, C. K.; Shin, F. G. Effect of Inclusion Deformation on the Magnetoelectric Effect of Particulate Magnetostrictive/Piezoelectric Composites J. Appl. Phys. 2007, 102, 063908.

47. Zhou, Y.; Shin, F. G. Magnetoelectric Effect of Mildly Conducting Magnetostrictive/Piezoelectric Particulate Composites J. Appl. Phys. 2006, 100, 043910. 48. Martins, P.; Caparros, C.; Goncalves, R.; Martins, P. M.; Benelmekki, M.; Botelho, G.; Lanceros-Mendez, S. Role of Nanoparticle Surface Charge on the Nucleation of the Electroactive Beta-Poly(Vinylidene Fluoride) Nanocomposites for Sensor and Actuator Applications J. Phys. Chem. C 2012, 116, 15790-15794.

49. Maceiras, A.; Martins, P.; Gonc, alves, R.; Botelho, G.; Venkata Ramana, E.; Mendiratta, S. K.; San Sebastián, M.; Vilas, J. L.; Lanceros-Mendez, S.; León, L. M. High-Temperature Polymer Based Magnetoelectric Nanocomposites Eur. Polym. J. 2015, 64, 224-228.

50. Martins, P.; Silva, M.; lanceros-mendez, s. Determination of the Magnetostrictive Response of Nanoparticles Via Magnetoelectric Measurements Nanoscale 2015, 21, 9457-9461.

Table of Contents Graphic and Synopsis

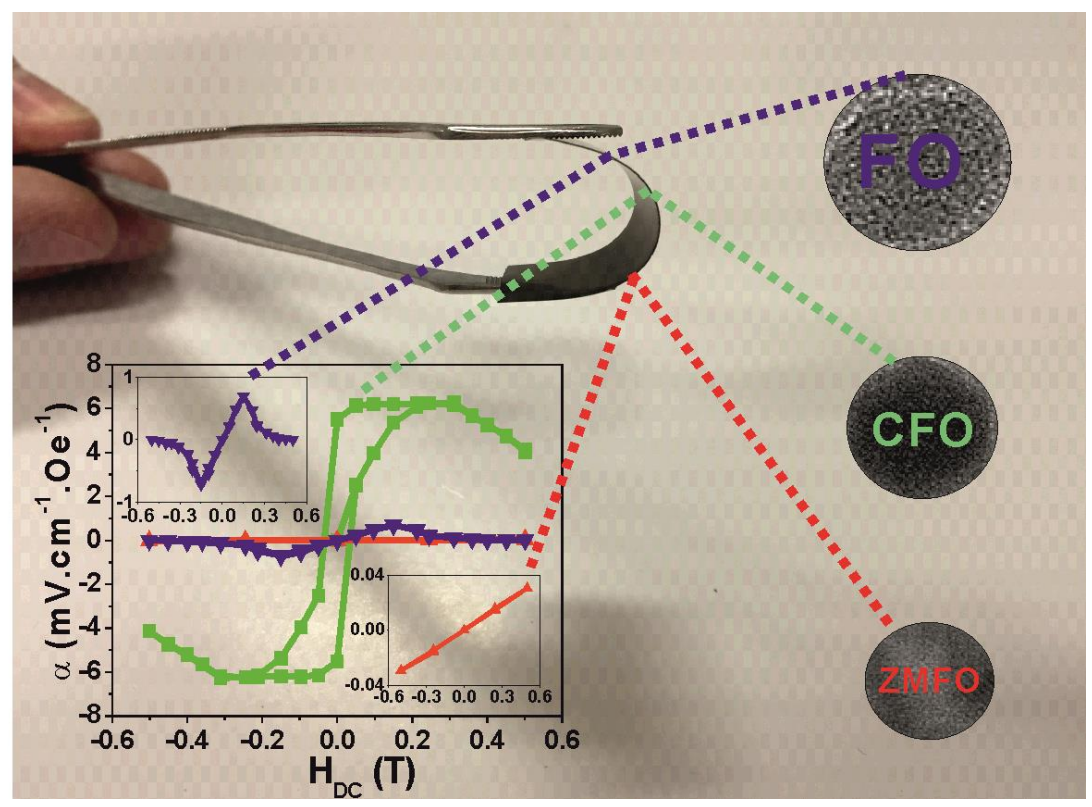

